\title{
Simulación de una Torre de Enfriamiento Mecánica Comparada con Curvas Experimentales
}

\author{
Jader D. Alean ${ }^{1}$, Gail A. Gutiérrez ${ }^{1}$, Farid Chejne ${ }^{2}$ y Marlon J. Bastidas ${ }^{2}$ \\ (1) Universidad Popular del Cesar, bloque A, oficina 205, sede Hurtado, salida a Patillal, \\ Valledupar, Cesar-Colombia (e-mail: ingenieroalean@gmail.com, gailgutierrez@unicesar.edu.co) \\ (2) Universidad Nacional de Colombia, Facultad de Minas, Instituto de Energía, Escuela de \\ Procesos y Energía, carrera 80 No. 65-223, Medellín, Antioquia-Colombia
}

\begin{abstract}
Resumen
El objetivo del trabajo es modelar y simular una torre de enfriamiento mecánica forzada a escala piloto. Las variables físicas se correlacionaron a partir de la transferencia de calor y materia y los resultados de la simulación son analizados mediante graficas que muestran la variación de la humedad, flujo de agua, calor latente, calor sensible, calor total, temperatura del agua y del aire. El coeficiente de transferencia de materia se obtuvo a partir de los datos experimentales y la solución numérica del modelo se obtuvo con el método Runge-Kutta en Matlab. La verificación de los resultados fue realizada, comparando las curvas simuladas con las curvas experimentales. Se concluye que la cercanía entre las curvas depende del coeficiente de transferencia de materia.
\end{abstract}

Palabras clave: torre de enfriamiento, humidificación, modelado, simulación, transferencia de calor y materia

\section{Simulation of a Mechanical Cooling Tower Compared with Experimental Curves}

\begin{abstract}
The objective of this work was the modeling and simulation of a pilot-scale mechanical enforced cooling tower. The physical variables were correlated from the heat and mass transfer and the simulation results were analyzed using graphs showing the change in humidity, water flow, latent heat, heat sensitive, total heat, water temperature and air. The mass transfer coefficient was obtained from experimental data and the numerical solution of the model was obtained using RungeKutta method in Matlab. Comparison between stimulation results and experimental data was done. It is concluded that the shape of the curves and the deviations of the simulated results depend on the mass transfer coefficient.
\end{abstract}

Keywords: cooling tower, humidification, modeling, simulation, heat and mass transfer 


\section{INTRODUCCIÓN}

En el ámbito industrial una torre de enfriamiento es utilizada como un intercambiador de calor, que enfría el agua caliente proveniente de un proceso, esto se realiza con el fin de recircular el agua y/o evitar la contaminación térmica por su vertimiento al ambiente. En la transferencia del calor en una torre de enfriamiento, existe generación de calor sensible y calor latente, al entrar en contacto el agua caliente con el aire. La masa de agua evaporada extrae el calor latente de vaporización del líquido, el cual es cedido al aire obteniéndose el enfriamiento del agua y el aumento de la temperatura del aire. A pesar que Walkel (1923) en su libro Principles of Chemical Engineering, fue el primero en proponer balances sencillos de masa y energía para torres de enfriamiento (Khan et al., 2003), es Merkel (1925) con su trabajo Zeitschrift des Vereines Deutscher Ingenieure, quien estructuró la teoría básica de estas (Khan et al., 2003). El modificó lo expuesto por Walker, relacionando el calor sensible y el calor latente en los balances de masa y energía. Webb (1989), en su trabajo Design of Cooling Towers by the Effectiveness-NTU Method, mostró que la eficiencia y las unidades de difusión en una torre, son consistentes con las usadas en otros intercambiadores de calor (citado en Khan et al., 2003). Nimr (1999), presentó un modelo que describe el comportamiento de una torre, para ello tuvo en cuenta el empaque o relleno en la torre. Milosavjevic (2001), determinó un modelo en ecuaciones diferenciales ordinarias, para describir la transferencia de masa y calor en una torre de enfriamiento mecánica, él utilizó datos experimentales en un equipo piloto para analizar con una simulación, el comportamiento de la torre con diferentes empaques. Kloppers y Kröger (2005a; 2005b), evaluaron el número de Lewis (relación entre el coeficiente de transferencia de masa y calor, con el calor húmedo) en una torre de enfriamiento y encontró que la relación disminuye, cuando el aire entra relativamente húmedo y caliente en la torre. Kloppers y Kröger (2005a; 2005b) plantearon un modelo matemático en ecuaciones diferenciales ordinarias para determinar la transferencia de masa y energía en una torre de enfriamiento, usó el método de Poppe para el calcular diferencialmente la integral de Merkel, la solución numérica la obtuvo con el método Runge-Kutta de cuarto orden y alcanzó buenos resultados. Fisenko y Brin (2007), describen la transferencia de masa y calor en una torre, acopla un modelo ecuaciones diferenciales parciales (para la temperatura del aire y del agua), con uno en ecuaciones diferenciales ordinarias (para el crecimiento de una gota de agua). Kranc (2007), desarrolló un modelo computacional para simulación, tuvo en cuenta la distribución no uniforme del agua en el empaque.

En los últimos años se han desarrollado nuevas herramientas en el modelo de torres de enfriamiento como la de Xiaoni (2007), quien desarrolló un modelo matemático en ecuaciones diferenciales ordinarias, él aplicó el concepto de Red Neuronal Artificial (ANA), proponiéndola como una herramienta de diseño computacional que ayuda a evaluar la temperatura de agua a la salida. Los resultados que predecía el ANA fueron comparados con los obtenidos del modelo matemático y con los datos experimentales. La ANA predijo la temperatura del agua con un margen de error absoluto de $1.31 \%$, y de $9.42 \%$ para el modelo. Ming Gao (2008) aplicó una ANA para predecir el funcionamiento de una torre, la ANA mostró un buen comportamiento estadístico, el coeficiente de correlación estaba en el rango de 0.993 - 0.999.

El objetivo del presente trabajo, es modelar y simular una torre de enfriamiento mecánica, con esta se pueden observar el comportamiento térmico mediante sistemas gráficos. La verificación del código, se realizó comparando los resultados de la simulación con los resultados experimentales obtenidos en una torre de enfriamiento a escala piloto. El modelo matemático se estructuró, tomando como base las ecuaciones diferenciales de calor latente y calor sensible, desarrollado por Milosavjevic (2001). El coeficiente de trasferencia de masa es obtenido por el método de integración directa, la relación de Lewis se asumió como uno, ya que el aire a la entrada de la torre no es húmedo y tampoco caliente (Kloppers y Kröger, 2005a; 2005b). Al igual que Milosavjevic (2001) y Kloppers y Kröger (2005a; 2005b), el modelo se solucionó con el método Runge-Kutta de paso variable. Las consideraciones físicas tenidas en cuenta, pueden resumirse como: La resistencia para la transferencia de calor en la película liquida es despreciable, el coeficiente de transferencia de masa, el calor del aire y del agua son constantes a lo largo de la sección trasversal, la temperatura de la interface entre el agua y el aire se asume igual a la temperatura del agua y se considera saturada, la columna de la torre opera adiabáticamente y la aproximación será mayor cuando aumenta el diámetro de la columna. 


\section{MODELO MATEMÁTICO Y SU ESTRUCTURA}

Las ecuaciones del modelo relacionan la transferencia de masa y calor en estado estable, derivadas respecto a la altura (Fig. 1). El sistema de ecuaciones, se resuelve con las condiciones de entrada del agua, del aire y con los siguientes parámetros: área de sección transversal $(A)$, altura $(Z)$ y coeficiente de transferencia de masa $\left(K_{G} a\right)$, este último es calculado con el método de integración directa. Aquí se propone el método Runge-Kutta (Chapra y Canale, 1999), para la solución numérica en MATLAB. La Ec. (1) representa la Variación de la humedad (Treybal, 1988), la Ec. (2) la variación del calor sensible (Milosavjevic, 2001), la Ec. (3) la variación del calor latente (Milosavjevic, 2001), la Ec. (4) la variación de la temperatura del aire, la Ec. (5) la variación de la temperatura del agua. Una deducción detallada del modelo es desarrollada por Milosavjevic (2001).

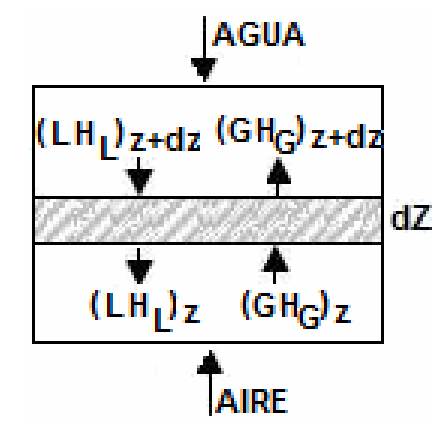

Fig. 1: Nomenclatura para una torre de enfriamiento (Milosavjevic, 2001)

El conjunto de ecuaciones diferenciales que pueden ser resueltas numéricamente, proveyendo las condiciones iniciales y los parámetros del modelo (área de sección transversal, A; altura de la torre, $\mathrm{Z}$; y coeficiente de transferencia de masa $\mathrm{K}_{\mathrm{G}} \mathrm{a}$ ), se muestran a continuación:

$$
\begin{aligned}
& \frac{d Y}{d Z}=\frac{h a}{C h} \frac{A}{G}\left(Y_{S}-Y\right) \\
& \frac{d Q_{S}}{d Z}=h a A\left(T_{L}-T_{G}\right) \\
& \frac{d Q_{L}}{d Z}=\left(\frac{h a h_{f g} A}{C h}\right)\left(Y_{S}-Y\right) \\
& \frac{d T_{G}}{d Z}=\frac{A\left[h a\left(T_{L}-T_{G}\right)+\frac{h a}{C h}\left(Y_{S}-Y\right) h_{f g}\right]}{G C h}-\frac{\left(h_{f g}+C_{V} T_{G}\right)}{C h} \frac{d Y}{d Z} \\
& \frac{d T_{L}}{d Z}=-\left[\frac{A\left[h a\left(T_{L}-T_{G}\right)+\frac{h a}{C h}\left(Y_{S}-Y\right) h_{f g}\right]}{C_{L} L}+\frac{G T_{L}}{L} \frac{d Y}{d Z}\right]
\end{aligned}
$$

\section{METODOLOGÍA}

Para alcanzar los objetivos desarrollados, el proyecto se estructuró y se organizó a través de una serie de fases con una secuencia metódica que abarcaron las etapas de conceptualización, modelado matemático teniendo en cuenta las variables físicas en una torre de enfriamiento (temperatura del agua, temperatura del aire, humedad, presión, coeficiente de transferencia de masa, flujos de agua y flujo de aire.), determinación del método numérico (Runge-Kutta) y simulación en MATLAB. La verificación del código computacional se realiza comparando los resultados experimentales, con los resultados que aquí se obtienen. Los datos experimentales fueron tomados en una torre de enfriamiento mecánica forzada a escala piloto, en ella, los puntos de medición son ocho (siete a lo largo de la sección transversal y uno en la parte superior de la torre), en cada punto se registraron mediciones de humedad relativa, temperatura del agua y temperatura del aire. 


\section{RESULTADOS Y DISCUSIÓN}

Después de obtener los resultados de la simulación, estos se compararon con los obtenidos experimentalmente de una torre piloto. Los datos de entrada en la simulación son: temperatura del agua, temperatura del aire, humedad relativa, flujo de agua, flujo de aire, coeficiente de transferencia de masa, altura y área de la torre (tabla 1). Los resultados a la salida, son las graficas de: temperatura del agua, temperatura del aire, humedad relativa, flujo de agua, calor latente, calor sensible y calor total. El modelo fue resuelto con la herramienta ode45 en MATLAB. La determinación del coeficiente de transferencia de masa jugó un papel importante, ya que de este depende la cercanía entre las curvas simuladas y reales (Fig. 2, 3, 4). El coeficiente de transferencia de masa fue obtenido con el método de Carey-Williamson y el método de integración (Kern, 1999). Las pequeñas diferencias, evidencian que el método de Carey-Williamson a pesar de ser utilizado industrialmente, acumula pequeños errores, ya que se calcula proyectando entalpías promedios y supone las tendencias en las líneas de operación. Se optó por el método de integración (tabla 2), con este los resultados son confiables, porque los datos de temperatura provienen de la toma directa de cada uno de los puntos en la sección transversal, denotando un comportamiento entálpico del equipo más exacto. Con el método de integración se obtuvieron diferentes coeficientes, al variar el flujo de agua y de aire, esto sirvió para realizar varias comparaciones, simuladas, con aceptables comportamientos. Aquí se tomaron, los datos para el punto óptimo de operación del equipo (0.1262 $\mathrm{Kg} / \mathrm{s}$ de agua y $0.0742 \mathrm{Kg} / \mathrm{s}$ de aire), permitiendo establecer el coeficiente global de transferencia en $1.4 \mathrm{Kg} / \mathrm{m}^{3} \mathrm{~s}$, valor que está dentro de lo estimado según curvas de rellenos comerciales.

Tabla 1: Resultados de la simulación y datos experimentales.

\begin{tabular}{|l|c|l|c|}
\hline \multicolumn{2}{|c|}{ DATOS DE ENTRADA } & \multicolumn{2}{c|}{ DATOS DE SALIDA } \\
\hline Flujo de aire entrante $(\mathrm{Kg} / \mathrm{s})$ & 0.0742 & \multicolumn{2}{c|}{ RESULTADOS SIMULACIÓN } \\
\hline Temperatura aire entrante $\left({ }^{\circ} \mathrm{C}\right)$ & 32 & Temperatura agua saliente $\left({ }^{\circ} \mathrm{C}\right)$ & 34.66 \\
\hline Temperatura Bulbo Húmedo entrante $\left({ }^{\circ} \mathrm{C}\right)$ & 26.4 & Temperatura aire saliente $\left({ }^{\circ} \mathrm{C}\right)$ & 34.66 \\
\hline Humedad relativa entrante $(\%)$ & 65 & Humedad Relativa saliente $(\%)$ & 96.71 \\
\hline Flujo del agua entrante $(\mathrm{Kg} / \mathrm{s})$ & 0.1262 & \multicolumn{2}{c|}{ DATOS REALES } \\
\hline Temperatura agua entrante $\left({ }^{\circ} \mathrm{C}\right)$ & 40.8 & Temperatura agua saliente $\left({ }^{\circ} \mathrm{C}\right)$ & 34.44 \\
\hline Área de la torre $\left(\mathrm{m}^{2}\right)$ & 0.0625 & Temperatura aire saliente $\left({ }^{\circ} \mathrm{C}\right)$ & 34.9 \\
\hline Altura de la torre $(\mathrm{m})$ & 1.42 & Humedad Relativa saliente $(\%)$ & 94.0 \\
\hline Coeficiente de transferencia de masa $\left(\mathrm{Kg} / \mathrm{m}^{3} \mathrm{~s}\right)$ & & \multicolumn{2}{c|}{1.4} \\
\hline Altura sobre el nivel del mar del sistema $(\mathrm{m})$ & \multicolumn{4}{|c|}{169} \\
\hline
\end{tabular}

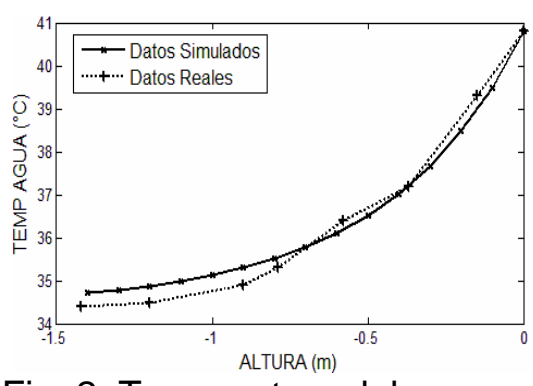

Fig. 2: Temperatura del agua

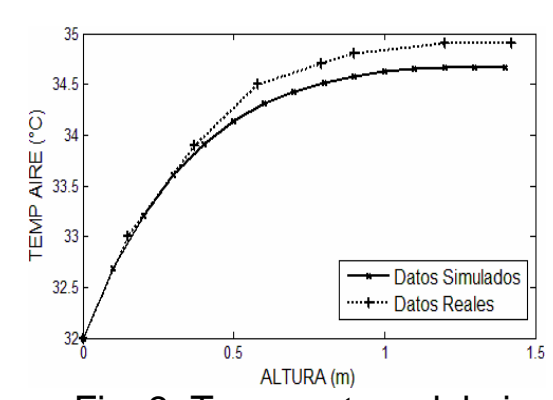

Fig. 3: Temperatura del aire

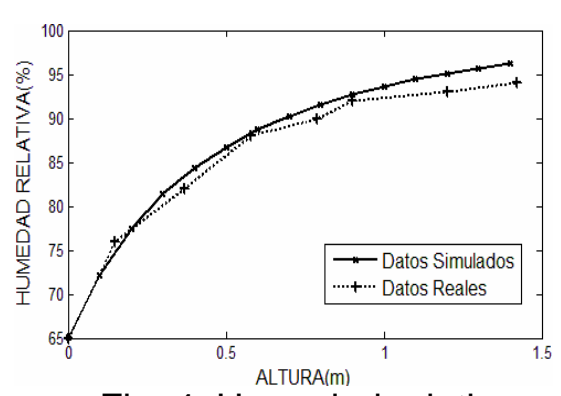

Fig. 4: Humedad relativa

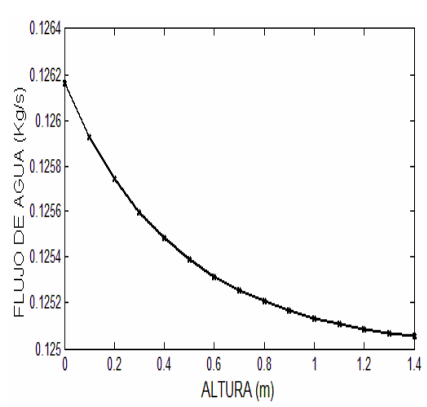

Fig. 5: Flujo de agua

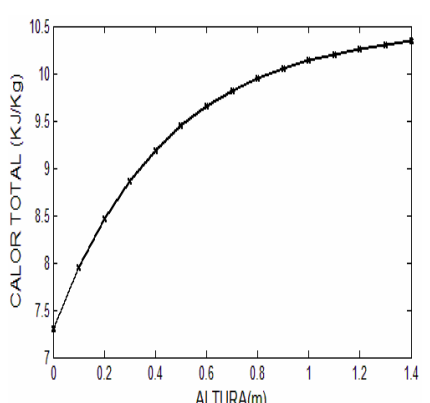

Fig. 6: Calor total

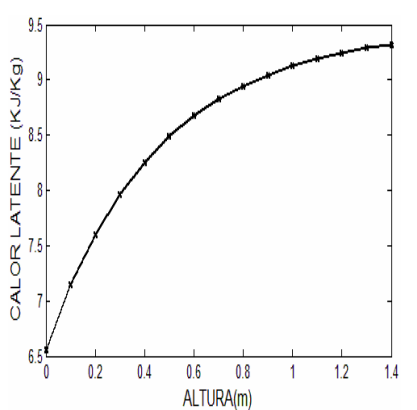

Fig. 7: Calor latente

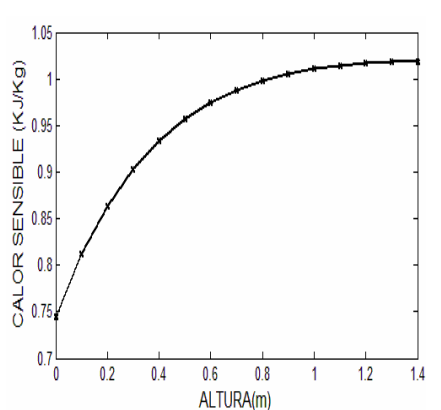

Fig. 8: Calor sensible 
Tabla 2: Determinación del coeficiente de transferencia de masa $\left(K_{G} a\right)$ por el método de integración

\begin{tabular}{|c|c|c|c|c|c|c|c|}
\hline$T_{L}$ & $H_{G_{S}}$ & $\mathrm{HR}$ & $H_{G}$ & $H_{G_{S}}-H_{G}$ & $\left(H_{G_{S}}-H_{G}\right)_{\text {promedio }}$ & $d T_{L}$ & $d T_{L} /\left(H_{G_{S}}-H_{G}\right)_{\text {promedio }}$ \\
\hline 34.4 & 127.50 & 65 & 93.50 & 34.0 & - & - & - \\
\hline 34.5 & 127.70 & 76 & 104.40 & 23.30 & 28.650 & 0.1 & 0.0035 \\
\hline 34.9 & 130.34 & 82 & 112.36 & 17.98 & 20.640 & 0.4 & 0.0194 \\
\hline 35.3 & 133.03 & 88 & 120.70 & 12.33 & 15.155 & 0.4 & 0.0264 \\
\hline 36.4 & 140.69 & 90 & 129.67 & 11.02 & 11.675 & 1.1 & 0.0942 \\
\hline 37.2 & 146.52 & 92 & 137.25 & 9.270 & 10.145 & 0.8 & 0.0788 \\
\hline 39.3 & 162.93 & 93 & 153.68 & 9.250 & 9.260 & 2.1 & 0.2268 \\
\hline 40.8 & 175.73 & 94 & 167.00 & 8.730 & 8.990 & 1.5 & 0.1668 \\
\hline \multicolumn{7}{|c|}{$K_{G} a A Z / C_{L} L=\int d T_{L} /\left(H_{G_{S}}-H_{G}\right)_{\text {promedio }}$} & 0.6159 \\
\hline \multicolumn{7}{|c|}{$K_{G} a$} & 1.4 \\
\hline
\end{tabular}

De la tabla 1, se deduce que la eficiencia de la torre a partir de la simulación es de $76.2 \%$, esta es obtenida relacionado la temperatura del agua de salida y la temperatura de bulbo húmedo, la eficiencia a partir de los datos reales es de $76.65 \%$, ambas eficiencias son buenas y es ratificado por la experiencia (se considera aceptable a partir del 70\%). Comparando la temperatura del agua a la salida, con la de la simulación, existe un $99.36 \%$ de proximidad. La mayor transferencia de calor en el agua ocurre a $-0.25 \mathrm{~m}$ (Fig. 2), lo equivalente a $1.17 \mathrm{~m}$ referenciando la parte inferior, aquí se enfría la mayor parte del agua y es donde existe la mayor transferencia de calor en el aire (Fig. 3). A los $1.42 \mathrm{~m}$ de altura se alcanza la humedad de salida correspondiente al $94 \%$ para el dato real y $96.71 \%$ para la simulación (Fig. 4), esto demuestra que la altura del equipo está dentro de lo estimando para enfriar el agua con las condiciones establecidas. En la Fig. 5 se deduce que 0.0015 $\mathrm{Kg} / \mathrm{s}$ corresponde al agua evaporada (0.95\%), de las Fig. 6, 7 y 8 se aprecia que el calor latente tiene mayor incidencia en el sistema.

\section{CONCLUSIONES}

Simular computacionalmente una torre de enfriamiento y compararla con datos experimentales, es necesario porque se puede ver la exactitud y veracidad de los resultados computacionales. Esto es importante, porque ayuda a facilitar la comprensión del proceso y académicamente es aplicable si no se cuenta con este equipo. El aporte más importante en este estudio, se relaciona con el análisis de los fenómenos de transferencia, la estructura del modelo y su relación con el coeficiente global de transferencia de masa. La simplificación más importante, fue considerar que el coeficiente global de transferencia de calor para el agua y el aire es igual, asumiendo que el todo el calor disipado por el agua es transferido al aire. Determinar el coeficiente global de transferencia de masa fue necesario, porque el empaque del equipo no es comercial y se observó que este, es crítico para ajustar con precisión el modelo. La verificación de los resultados computacionales, se realizó comparando los resultados experimentales tomados en una torre piloto, con los de la simulación, esto indicó un buen comportamiento de la misma y mostrando que una pequeña cantidad de agua evaporada, produce grandes efectos de enfriamiento.

\section{AGRADECIMIENTOS}

A Colciencias, a la Universidad Popular del Cesar y a la Universidad Nacional de Colombia.

\section{NOMENCLATURA}

A Área de sección transversal de la torre $\left(\mathrm{m}^{2}\right)$.

a Superficie de transferencia por unidad de volumen $\left(\mathrm{m}^{2} / \mathrm{m}^{3}\right)$.

$C_{G} \quad$ Calor específico del aire seco $\left(\mathrm{KJ} / \mathrm{Kg}^{\circ} \mathrm{C}\right)$

$C_{h} \quad$ Calor húmedo $\left(\mathrm{KJ} / \mathrm{kg}^{\circ} \mathrm{C}\right)$.

$C_{L} \quad$ Calor específico del agua $\left(\mathrm{KJ} / \mathrm{Kg}^{\circ} \mathrm{C}\right)$

$C_{V} \quad$ Calor específico del vapor de agua $\left(\mathrm{J} / \mathrm{Kg}^{\circ} \mathrm{C}\right)$

$\dot{G} \quad$ Flujo de aire seco (kg/s). 
ha Coeficiente global de transferencia de calor por unidad de volumen $\left(\mathrm{KJ} / \mathrm{m}^{3 \circ} \mathrm{C} \mathrm{s}\right)$.

$H_{G} \quad$ Entalpía del aire $(\mathrm{KJ} / \mathrm{Kg})$

$H_{G_{S}} \quad$ Entalpía del aire saturado $(\mathrm{KJ} / \mathrm{Kg})$

HR Humedad Relativa (\%)

$h_{f g} \quad$ Calor latente de vaporización $(\mathrm{KJ} / \mathrm{kg})$.

$\dot{L} \quad$ Flujo de agua $(\mathrm{kg} / \mathrm{s})$.

$\dot{Q}_{L} \quad$ Flujo de calor latente $(\mathrm{KJ} / \mathrm{s})$

$\dot{Q}_{s} \quad$ Flujo de calor sensible $(\mathrm{KJ} / \mathrm{s})$

$\mathrm{T}_{\mathrm{L}} \quad$ Temperatura del agua $\left({ }^{\circ} \mathrm{C}\right)$

$\mathrm{T}_{\mathrm{G}} \quad$ Temperatura del aire $\left({ }^{\circ} \mathrm{C}\right)$

$Y \quad H u m e d a d$ absoluta del aire (kg agua/kg gas seco).

$Y_{S} \quad$ Humedad absoluta del aire saturado ( $\mathrm{kg}$ agua/kg gas seco).

$\mathrm{Z} \quad$ Altura de la torre $(\mathrm{m})$.

\section{REFERENCIAS}

Chapra, S y R. Canale; Métodos Numéricos para Ingenieros, 550- 564, McGraw-Hill, Ciudad de México, México (1999).

Fisenko, S.P y A.A. Brin; Simulation of a cross-flow cooling tower performance, International Journal of Heat and Mass Transfer: 50, 3216-3218 (2007).

Kern, D.; Procesos de transferencia de calor, 689-690, CEDSA, Ciudad de México, México (1999).

Khan, J., M. Yaqub y A.M. Zubair; Performarce Characteristics of Counter Wet Cooling Towers, International Journal of Energy Conversion and Management: 44, 2073-2091 (2003).

Kloppers, J y D.G. Kröger; The Lewis factor and its influence on the performance prediction of wetcooling towers, International Journal of Thermal Sciences: 44, 879-884 (2005a).

Kloppers, J y D.G. Kröger; A critical investigation into the heat and mass transfer analysis of counterflow wet-cooling towers, International Journal of Heat and Mass Transfer: 48, 765-777 (2005b).

Kranc, S.; Optimal spray patterns for counterflow cooling towers with structured packing, Applied Mathematical Modeling: 31, 676-686 (2007).

Milosavlevic, N.; A comprehensive approach to cooling tower design, International Journal of Applied Energy: 21, 901-903 (2001).

Ming Gao., A.; Performance prediction of wet cooling tower using artificial neural network under cross-wind conditions, International Journal of Thermal Sciences: 1-7 (2008)

Nimr, M.; Modeling the dynamic thermal behavior of cooling towers containing packing materials, Heat Transf. Eng.: 20, 91-96 (1999).

Treybal, R.; Operaciones de Transferencia de Masa. $2^{a}$ edición, 275-278, McGraw-Hill, México (1988).

Xiaoni, Q., L. Zhenyan y L. Dandan; Numerical simulation of shower cooling towers based on artificial neural network, Energy Conversion and Management: 49(4), 724-732 (2008). 